\title{
Time on therapy and concomitant medication use of mepolizumab in Canada: a retrospective cohort study
}

\section{To the Editor:}

Asthma is a chronic inflammatory condition that affects 3.8 million Canadians and nearly 65000 acute asthma exacerbations occur each year [1-4]. Left untreated, asthma can lead to increasing mortality and morbidity $[2,5,6]$. Economically, the projected 20-year total costs (2014-2033) of suboptimal asthma control in Canada are estimated to be $\$ 213$ billion, of which the majority ( $\$ 195$ billion) were productivity losses (presenteeism and absenteeism) [7].

In Canada and abroad, asthma control and the prevention of exacerbations is a treatment priority [3]. Current international guidelines advise the use of a combination inhaled corticosteroid (ICS) and long-acting $\beta_{2}$-agonist (LABA) (specifically formoterol) for patients with mild disease (Global Initiative for Asthma (GINA) steps 1-2), progressing to daily use of ICS/LABA according to the severity of the disease (GINA step 3-5), and recommending the addition of tiotropium, anti-IgE or anti-interleukin-5 biologics for patients with more severe disease (GINA step 5) $[5,6]$.

Approximately $7 \%$ of patients with asthma are estimated to have severe uncontrolled disease, leading to substantial health impact as these patients contribute $>60 \%$ of asthma-related healthcare costs [8]. Biologic therapies provide effective treatment options that may improve asthma control for these patients and Health Canada has approved several for the treatment of patients with severe asthma. Despite widespread clinical use and increasing availability, little is known about the real-world clinical outcomes related to continued therapeutic use for several of these biologic therapies.

At the time of this analysis, mepolizumab (NUCALA, GlaxoSmithKline) has gained private and public market access across Canada and accumulated a cohort of patients with sufficient follow up to perform this study. We evaluated the real-world time to discontinuation of mepolizumab using Canadian claims data, controlling for available patient factors (biologic treatment history, sex, age and region), and the concomitant use of short-acting $\beta_{2}$-agonists (SABA) and oral corticosteroids (OCS).

Data were extracted from the IQVIA Private Drug Plan (PDP) dataset, which contains deidentified patient-level data captured from 12 million active claimants (2012) across Canada, representing $~ 80 \%$ of the entire private market. All sources of data are actively managed, quality-controlled and capture patient demographic characteristics, the specific drug dispensed, quantity dispensed, number of days supplied and service date. This is a secondary analysis of anonymised administrative data and, therefore, ethics approval was not required.

All patients $\geqslant 18$ years of age in the PDP database were eligible if they made at least one claim for mepolizumab between 1 March 2016 and 20 December 2019. Participants were excluded if their age or sex was missing and had $<3$ months drug plan history prior to initiation of therapy. Participants were followed until either mepolizumab discontinuation or the end of the follow-up period, at which point data were censored.

$@$ ERSpublications

About half of the patients who initiate therapy with mepolizumab discontinue treatment within the first or second year. Concomitant use of short-acting $\beta_{2}$-agonists and oral corticosteroids drops during mepolizumab use. https://bit.ly/3aRISqS

Cite this article as: Lee $\mathrm{JK}$, Gendron $\mathrm{A}$, Knutson $\mathrm{M}$, et al. Time on therapy and concomitant medication use of mepolizumab in Canada: a retrospective cohort study. ERJ Open Res 2021; 7: 00778-2020 [https://doi.org/10.1183/23120541.00778-2020]. 
The primary outcome was time to treatment discontinuation, measured as the difference between date of final claim and date of first claim for mepolizumab. Date of final claim was defined as the latest chronological claim, with no further claims for mepolizumab for 90 days while maintaining drug plan status. Patients were censored if they had insufficient data to determine future claims (i.e. did not have 90 days of follow-up or drug plan activity after the final claim).

We captured age (years), sex (male or female), region of origin (British Columbia, Alberta, Prairies (Saskatchewan and Manitoba), Ontario, Quebec or Atlantic (New Brunswick, Nova Scotia, Newfoundland or Prince Edward Island)) and biologics treatment history (any claims for biologics in the 3-12 months prior to mepolizumab initiation).

Categorical data were summarised as $\mathrm{n}(\%)$ and continuous data were summarised as mean $\pm \mathrm{sD}$. We ran a Cox regression model to determine the effect of covariates on risk of discontinuation. We adjusted for age, sex, province and treatment history with biologics, and performed a sensitivity analysis based on year of initiation. Time to discontinuation was computed using the Kaplan-Meier method. We used ANOVA to investigate the difference in the average proportion of people with claims made before, during and after mepolizumab use. Differences between these three periods were tested accounting for multiple comparisons using the Games-Howell procedure.

Data were available for 1441 patients. The mean age was $52 \pm 11.2$ years. Close to half of the participants were female (55.2\%). Mean follow-up time was $11.6 \pm 9.5$ months (median 9 months, interquartile range 4-17 months). Almost half were from the province Ontario $(683,47.4 \%)$ and $13 \%$ (196) had a prior history of treatment with biologics.

At 12 months post-treatment initiation, $40.3 \%$ of patients discontinued treatment with mepolizumab ( $95 \%$ CI $37.3-43.1 \%)$, increasing to $57.6 \%(95 \%$ CI $54.0-60.9 \%)$ at 24 months. The mean time to discontinuation was $21.5 \pm 0.5$ months (median 19 months, interquartile range 17-22 months) (figure 1a). Males were less likely to discontinue treatment than females (adjusted hazard ratio 1.18, 95\% CI 1.01-1.39; $\mathrm{p}=0.047$ ). Monthly utilisation of SABA and OCS are shown in figure $1 \mathrm{~b}$, suggesting decreased use of OCS or SABA while on mepolizumab, in line with previous trials.

In this cohort of adult Canadians with severe asthma, we found that $40.1 \%$ and $54.6 \%$ of patients had discontinued mepolizumab treatment at 12 and 24 months, respectively. Males were more likely to discontinue than females. The use of SABA or OCS significantly decreased relative to the pre-treatment periods but the magnitude of this benefit was not sustained following discontinuation (figure 1b), in line with results from other studies $[9,10]$.

To the best of our knowledge, this is the first report on real-world use of mepolizumab in the Canadian setting. In Canada, treatment with mepolizumab requires special access authorisation with rigid criteria for initial and continued reimbursement. Therefore, discontinuation rates within this cohort are a relevant outcome for payers interested in system efficiency and therapeutic value, and for physicians who may see this as a surrogate for treatment response $[9,10]$. These rates are also much higher than those in clinical trials, which noted $\sim 14 \%$ discontinuation in the first year [11].

a)

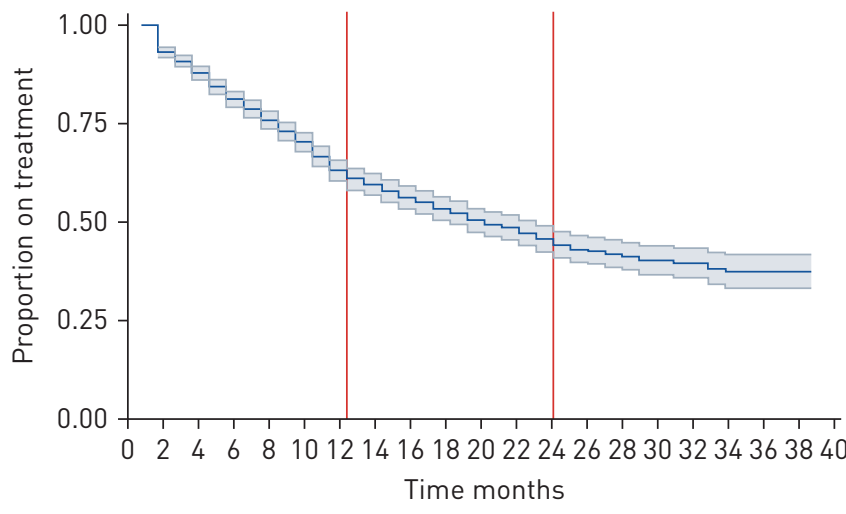

b)

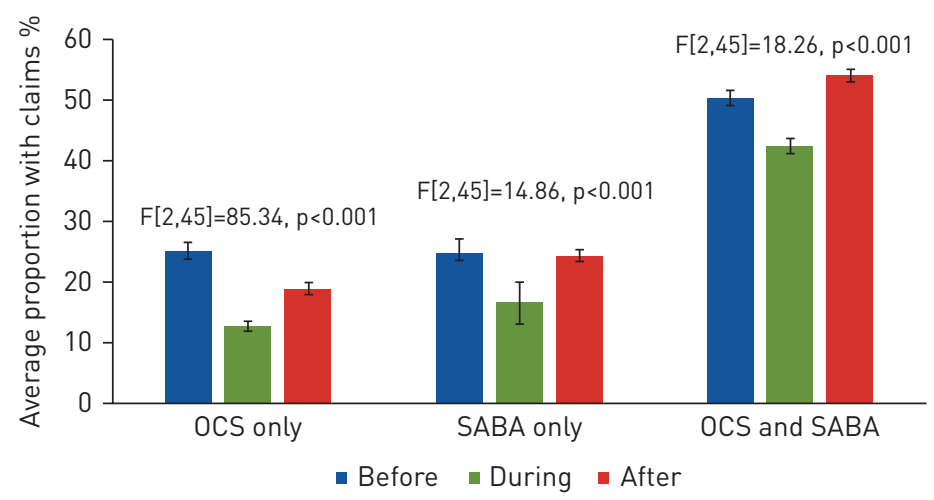

FIGURE 1 a) Kaplan-Meier survival curve for discontinuation. b) Trends in average monthly claims for rescue medications before, during and after use of mepolizumab. Bars represent means and error bars represent standard deviations. OCS: oral corticosteroid; SABA: short-acting $\beta_{2}$-agonist. F-tests were performed for differences between groups. 
However, several important limitations are present, given the nature of this database. Clinically, these data do not detail the reason for discontinuation and thus the direction (positive or negative) of the treatment response $[9,10]$. In addition, although we included only those patients who are actively using their private medical insurance, we must consider the impact of manufacturer-associated patient access programmes (e.g. reimbursement bridging programmes) relative to our use of the date of first reimbursement claim as an indication of treatment initiation. Nonetheless, sensitivity analyses of a subset of patients selected from a period with high reimbursement coverage and low access support showed no difference in discontinuation from the overall cohort. Finally, utilisation of SABA and OCS data were only available in aggregate form, which precludes our ability to investigate the relationship of these covariates with the discontinuation outcomes.

In summary, $40 \%$ of the patients that initiate therapy with mepolizumab discontinue treatment within the first year of treatment and $55 \%$ by the end of the second year of therapy. This level of discontinuation suggests substantial waste of time and resources given the cost of the drug and the clinician time involved in reimbursement navigation. Importantly, for both patients and care providers, we cannot rule out clinical reasons for discontinuation, including adverse events, treatment failure, suboptimal response and patient preference [12]. Patients may also face challenges in securing time away from work for injections.

Jason K. Lee ${ }^{1}$, Alain Gendron $\oplus^{2,3}$, Michelle Knutson ${ }^{4}$, Niroshan Sriskandarajah ${ }^{2}$, Lawrence Mbuagbaw $\odot^{5}$ and Stephen G. Noorduyn ${ }^{2}$

${ }^{1}$ Evidence Based Medical Educator, Toronto, Canada. ${ }^{2}$ AstraZeneca, Mississauga, Canada. ${ }^{3}$ Dept of Medicine, University of Montreal, Quebec, Canada. ${ }^{4}$ University of Toronto, Toronto, Canada. ${ }^{5}$ Dept of Health Research Methods, Evidence and Impact, McMaster University, Hamilton, Canada.

Correspondence: Stephen G. Noorduyn, Medical Evidence Team, Scientific Affairs, AstraZeneca Canada, 1004 Middlegate Road, Mississauga, ON, L4Y 1M4, Canada. E-Mail: Stephen.noorduyn@astrazeneca.com

Received: 23 Oct 2020 | Accepted: 31 Jan 2021

Author contributions: J.K. Lee, A. Gendron, M. Knutson, N. Sriskandarajah and S.G. Noorduyn conceived of the study. L. Mbuagbaw, M. Knutson and S.G. Noorduyn supported the design of the study. S.G. Noorduyn and M. Knutson collected data. M. Knutson and L. Mbuagbaw analysed qualitative and quantitative data. L. Mbuagbaw wrote the first draft. J.K. Lee, A. Gendron, M. Knutson, N. Sriskandarajah and S.G. Noorduyn reviewed several versions of the manuscript. All authors reviewed and approved the final version.

Conflict of interest: J.K. Lee reports clinical research payments from AstraZeneca during the conduct of the study; and clinical research payments, speaker fees and patient support programmes from AstraZeneca, GSK, Novartis, Regeneron, Sanofi, Takeda and Pfizer, clinical research for Genentech and Roche, speaker fees from Merck and Mylan, and an advisory board for Abbvie, outside the submitted work. A. Gendron reports employment with AstraZeneca Canada both during the conduct of the study and outside the submitted work. M. Knutson reports employment with AstraZeneca Canada both during the conduct of the study and outside the submitted work. N. Sriskandarajah reports employment with AstraZeneca Canada both during the conduct of the study and outside the submitted work. L. Mbuagbaw reports personal fees from AstraZeneca during the conduct of the study. S.G. Noorduyn reports employment with AstraZeneca Canada both during the conduct of the study and outside the submitted work.

Support statement: This work was funded by AstraZeneca. Funding information for this article has been deposited with the Crossref Funder Registry.

\section{References}

1 Public Health Agency of Canada. Asthma and Chronic Obstructive Respiratory Disease (COPD) in Canada. https:/www.canada.ca/en/public-health/services/publications/diseases-conditions/asthma-chronic-obstructivepulmonary-disease-canada-2018.html. Date last accessed: 19 October 2020. Date last updated: 9 December 2019.

2 Public Health Agency of Canada. Asthma in Canada. https://infobase.phac-aspc.gc.ca/datalab/asthma-blog-en. html. Date last accessed: 19 October 2020. Date last updated: 1 May 2018.

3 Public Health Agency of Canada. Fast facts about asthma: data compiled from the 2011 survey on living with chronic diseases in Canada. https://www.canada.ca/en/public-health/services/chronic-diseases/chronic-respiratorydiseases/fast-facts-about-asthma-data-compiled-2011-survey-on-living-chronic-diseases-canada.html. Date last accessed: 19 October 2020. Date last updated: 23 July 2015.

4 CIHI. Emergency department (ED) visits: volumes and median length of stay by triage level, visit disposition, and main problem. http://indicatorlibrary.cihi.ca/display/HSPIL/Asthma+Emergency+Department+Visits\%3A+Volume +and+Median+Length+of+Stay. Date last accessed: 19 October 2020. Date last updated: 2019.

5 Reddel HK, Bateman ED, Becker A, et al. A summary of the new GINA strategy: a roadmap to asthma control. Eur Respir J 2015; 46: 622-639.

6 Lougheed MD, Lemiere C, Dell SD, et al. Canadian Thoracic Society asthma management continuum - 2010 consensus summary for children six years of age and over, and adults. Can Respir J 2010; 17: 15-24.

7 Zafari Z, Sadatsafavi M, Chen W, et al. The projected economic and health burden of sub-optimal asthma control in Canada. Respir Med 2018; 138: 7-12. 
8 Sadatsafavi M, Lynd L, Marra C, et al. Direct health care costs associated with asthma in British Columbia. Can Respir J 2010; 17: 74-80.

9 Ke X, Kavati A, Wertz D, et al. Real-world clinical characteristics, treatment patterns, and exacerbations in US Patients with asthma newly treated with omalizumab. Clin Ther 2018; 40: 1140-1158.e1144.

10 Chen H, Eisner MD, Haselkorn T, et al. Concomitant asthma medications in moderate-to-severe allergic asthma treated with omalizumab. Respir Med 2013; 107: 60-67.

11 Pavord ID, Korn S, Howarth P, et al. Mepolizumab for severe eosinophilic asthma (DREAM): a multicentre, double-blind, placebo-controlled trial. Lancet 2012; 380: 651-659.

12 Mukherjee M, Forero DF, Tran S, et al. Sub-optimal treatment response to anti-IL-5 monoclonal antibodies in severe eosinophilic asthmatics with airway autoimmune phenomena. Eur Respir J 2020; 56: 2000117. 